\title{
The incidence of tuberculosis among adolescents and young adults: a global estimate
}

\author{
Kathryn J. Snow $0^{1,2}$, Charalambos Sismanidis ${ }^{3}$, Justin Denholm ${ }^{4,5}$, \\ Susan M. Sawyer ${ }^{6,7,8}$ and Stephen M. Graham ${ }^{1,9}$
}

Affiliations: ${ }^{1}$ Centre for International Child Health, University of Melbourne Dept of Paediatrics and Murdoch Children's Research Institute, The Royal Children's Hospital, Melbourne, Australia. ${ }^{2}$ School of Population and Global Health, University of Melbourne, Melbourne, Australia. ${ }^{3}$ Tuberculosis Monitoring and Evaluation Team, Global Tuberculosis Programme, World Health Organization, Geneva, Switzerland. ${ }^{4}$ Victorian Tuberculosis Program, Melbourne Health, Melbourne, Australia. ${ }^{5}$ Dept of Microbiology and Immunology, University of Melbourne, Melbourne, Australia. ${ }^{6}$ Centre for Adolescent Health, The Royal Children's Hospital, Melbourne, Australia. ${ }^{7}$ Murdoch Children's Research Institute, Melbourne, Australia. ${ }^{8}$ Dept of Paediatrics, The University of Melbourne, Melbourne, Australia. ${ }^{9}$ International Union Against Tuberculosis and Lung Disease, Paris, France.

Correspondence: Kathryn J. Snow, Centre for International Child Health, University of Melbourne Dept of Paediatrics, The Royal Children's Hospital, 50 Flemington Road, Parkville, Victoria 3052, Australia.

E-mail: kathryn.snowdunimelb.edu.au

@ERSpublications

An estimated 1.78 million young people (aged 10-24 years) around the world developed tuberculosis in $2012 \mathrm{http}: / /$ ow.ly/o9vq30hoepv

Cite this article as: Snow KJ, Sismanidis C, Denholm J, et al. The incidence of tuberculosis among adolescents and young adults: a global estimate. Eur Respir J 2018; 51: 1702352 [https://doi.org/10.1183/ 13993003.02352-2017].

ABSTRACT Historical data show that the risk of tuberculosis increases dramatically during adolescence, and young people face unique challenges in terms of case detection and effective treatment. However, little is known about the burden of tuberculosis among young people in the modern era. This study aimed to provide the first estimates of the global and regional incidence of tuberculosis among young people aged $10-24$ years.

Using the World Health Organization (WHO) database of tuberculosis notifications for 2012, we estimated the burden of tuberculosis among young people by WHO region. Adjustments were made for incomplete age disaggregation and underreporting, using supplementary data from several countries representing diverse tuberculosis epidemics.

We estimate that 1.78 million (uncertainty interval (UI) $1.23-3.00$ million) young people developed tuberculosis in 2012, accounting for $17 \%$ of all new tuberculosis cases globally. Young people in the WHO South East Asian Region (721000, UI 473000-1.35 million) and the WHO African Region (534000, UI 359000-912 000) experienced the greatest number of tuberculosis episodes.

Young people suffer a considerable burden of tuberculosis. Age-specific burden of disease estimation for this age group is complicated by incomplete age disaggregation of tuberculosis data, highlighting the importance of continued surveillance system strengthening. 


\section{Introduction}

Adolescence is increasingly recognised as a period of development that underpins many aspects of future health and well-being $[1,2]$. The success of the child survival agenda within the Millennium Development Goals (2000-2015), among other factors, has resulted in many countries now having more adolescents (10-19 years) and young adults (20-24 year olds) than younger children; young people aged 10-24 years now comprise a quarter of the world's population [3].

Adolescence and early adulthood is increasingly recognised as a key risk period for tuberculosis infection, disease and adverse outcomes. In contrast to young children aged 0-4 years, young people commonly develop infectious forms of tuberculosis and frequently have a much wider range of social contacts outside of the household [4]. Consequently, as well as suffering disease, adolescents and young adults with tuberculosis contribute to ongoing transmission. In high tuberculosis transmission settings, the incidence of tuberculosis increases rapidly during adolescence to peak in early adulthood [5]. Historical data suggest that the risk of infection with Mycobacterium tuberculosis is highest during adolescence and young adulthood, and that between the ages of 12 and 24 years there may also be a transient increase in the risk of progression to disease after infection compared with children or older adults $[5,6]$. Furthermore, many comorbidities relevant to tuberculosis emerge or are exacerbated during the adolescent period, including infection with HIV, diabetes, risky substance use (including tobacco use) and mental health conditions. Finally, many adolescent girls and young women face the health challenges associated with pregnancy and childbirth, which may increase their risk of both developing tuberculosis and experiencing adverse outcomes $[7,8]$.

Adolescents face specific age-related challenges in accessing appropriate care as they transition between child and adult health services, particularly in tuberculosis-endemic settings where dedicated adolescent health services are usually absent [1,2]. A study of adolescents aged 12-18 years in Kenya reported that their prevalence of tuberculosis was six-fold higher than indicated by case notifications [9]. A recent study from Botswana reported higher rates of loss to follow-up in 10-19-year-old adolescents on treatment for tuberculosis than among adults [10]. Adolescents and young adults are a recognised key population in the global HIV epidemic and HIV-related deaths are increasing among adolescents, in contrast to falling mortality in every other age group [11]. Although the importance of the adolescent age group in HIV control is increasingly appreciated, tuberculosis among adolescents remains neglected.

This at-risk age group requires increased attention for case detection, care and prevention within the global End TB Strategy [12]. A critical first step to addressing the issue of tuberculosis in at-risk groups is to "know your epidemic" [13]. The World Health Organization (WHO) first published estimates of the burden of tuberculosis in "children" (children and young adolescents aged 0-14 years) only as recently as 2012 [14]. In recognition of the limitations of data availability and interpretation, there have been ongoing efforts to improve the accuracy of these estimates using various statistical and modelling approaches [1517]. It is currently estimated that there were 1.0 million incident cases of tuberculosis and 210000 tuberculosis-related deaths in children and young adolescents in 2015 [17].

In contrast, despite being a highly dynamic period for health in general and tuberculosis in particular, there are no published estimates of tuberculosis incidence among adolescents and young adults globally. The majority of the literature on tuberculosis divides the population into two age groups, 0-14 years (referred to as "children") and $\geqslant 15$ years (referred to as "adults"), ignoring adolescents and young adults as a distinct group. The aim of this study was to estimate the global incidence of tuberculosis among young people aged 10-24 years in 2012 from the global tuberculosis notification dataset.

\section{Methods}

\section{Data sources}

While a majority of national tuberculosis programmes routinely report all notified child tuberculosis cases (0-14 years) each year to the WHO, and increasingly disaggregate data into the age groups of 0-4 and 5-14 years, the adolescent age group remains obscured by current reporting practices. Young adolescents (10-14 years) are included in the "child" age group, while older adolescents and young adults aged 15-24 years are categorised as "adults" [18]. This does not allow a direct accounting of tuberculosis notifications for adolescents and young adults aged 10-14, 15-19 and 20-24 years or a direct observation of the epidemiological changes that occur with tuberculosis across these years. In order to estimate the

Support statement: K.J. Snow is supported by the Australian National Health and Medical Research Council's Centre for Research Excellence in Tuberculosis, which funded this work. The funder had no role in the design or conduct of the study. Funding information for this article has been deposited with the Crossref Funder Registry.

Conflict of interest: None declared. 
global incidence of tuberculosis in adolescents and young adults, this study used the WHO global tuberculosis surveillance dataset for 2012 [19], supplemented with more detailed surveillance data from Brazil, Indonesia, South Africa, Romania and Estonia, disaggregated into 5-year age groups. These countries were chosen based on both data availability and epidemiological diversity. The analysis was restricted to 183 countries that reported notification data from 2012 to the WHO in 2013 and for which the United Nations Population Division provides age-disaggregated population estimates (in 10-year age groups).

Tuberculosis data for 2012 were used as that was the most recent completed dataset available at the outset of this project, and changes to the WHO recording and reporting framework for tuberculosis with the 2013 revision [20] prevent the application of the same estimation method presented in this article to more recent data on tuberculosis case notifications.

This analysis was exempt from ethical review as it utilised routinely collected, aggregated data only.

\section{Key variables}

Six key notification variables in the global tuberculosis surveillance dataset were used in this analysis: the numbers of notifications for each of 1) sputum smear-positive tuberculosis, 2) smear-negative (including smear not done) tuberculosis and 3) extrapulmonary tuberculosis, for each of the 0-14- and 15-24-year-old age groups. Where a value was missing for a specific country, feasible potential values were estimated based on available data as described in detail later. The resulting actual or estimated numbers of notifications for each country were then disaggregated into 5-year age bands $(0-4,5-9,10-14,15-19$ and 20-24 years) based on the observed distributions by age in the supplementary data. The resulting estimates were then adjusted for underreporting and underdiagnosis based on the estimates of case detection rates (CDRs) available for each country for 2016. CDR estimates from 2016 were used as these have undergone important revisions in recent years for some high-burden countries, with improved accuracy following national prevalence surveys, and these revisions apply retrospectively [17].

Analyses were conducted in Stata version 13 (StataCorp, College Station, TX, USA). Uncertainty intervals (UIs) were generated using data simulation bootstrapping techniques, generating a dataset with 1000 iterations of each country, assuming the key variables followed the distributions given in table 1. Each step of the process is now described in detail, with the relevant equations provided at the end of this section.

\section{Estimation of incidence among young adolescents aged 10-14 years}

The region-specific estimates for child tuberculosis were used to estimate the number of incident tuberculosis cases among 10-14 year olds, based on the observed proportions of notifications across countries in the supplementary dataset $(0.206$, UI $0.107-0.426)$. The methods used for estimation of the child tuberculosis burden by the WHO are described in detail in Annex 1 of the 2016 Global TB Report [17]. Estimates for 2016 rather than 2012 were used as, like CDRs, child tuberculosis estimates have been subject to important revisions in recent years, which were applied retrospectively to 2012 for the purposes of this analysis. The equation for young adolescent tuberculosis in each region was:

$$
\mathrm{TB}_{10-14}=\mathrm{TB}_{0-14} \times \mathrm{P}_{10-14}
$$

where $\mathrm{P}_{10-14}$ is the proportion of notifications among 0-14 year olds contributed by the 10-14-year-old age group. Child tuberculosis estimates are already adjusted for underreporting, removing the need for further adjustment for CDRs in this step.

\section{Disaggregation of the 15-24-year-old age group into 15-19 and 20-24 years}

The numbers of tuberculosis cases in the 15-19- and 20-24-year-old age groups were estimated at the country level using a three-step process. First, the number of sputum smear-positive notifications for the 15-24-year-old age group in each country was extracted from the global surveillance dataset. Where this value was missing for a country, it was estimated from the number of all new smear-positive notifications and the age structure of the population in the country, obtained from published United Nations Population Division estimates [3]. A linear model was used to estimate the proportion of all smear-positive cases that affect the 15-24-year-old age group, using the proportion of the national population aged 15-24 years as the explanatory variable. This estimation was necessary for seven countries with a combined total population of 156 million people (a large majority in Ethiopia, Peru and Mozambique; supplementary table S1).

Second, the numbers of notifications for smear-negative and extrapulmonary tuberculosis were extracted from the dataset. These variables were considered together because, when missing, they were almost always missing concurrently (the number of missing values for the key variables is summarised by country in 
supplementary table S1). Where the number of smear-negative and/or extrapulmonary tuberculosis notifications for the 15-24-year-old age group was not reported, the number was estimated based on the observed ratio of smear-negative plus extrapulmonary notifications to smear-positive tuberculosis notifications in the global dataset. This estimation was necessary for 50 countries (supplementary table S1).

Third, the proportion of all tuberculosis notifications from the 15-24-year-old age group that pertained to the 15-19-year-old age group was estimated from the observed proportions in the supplementary data; the median value was used for the point estimates and the two extreme values for the uncertainty interval. The remainder of the actual or estimated notifications were assumed to pertain to the 20-24-year-old age group.

\section{Accounting for underreporting of tuberculosis}

We adjusted actual and estimated notifications to adjust for underdetection and underreporting of tuberculosis using the reciprocal of the estimated CDR for each country as estimated in 2016 [21], with uncertainty incorporated during the bootstrapping process. This step was necessary for all countries.

\section{Accounting for uncertainty}

Each of the estimated parameters described was randomly generated for each of the 1000 iterations of each country in the bootstrapping process and allowed to vary according to the distributions listed in table 1. The normal distribution was used for the ratios and proportions derived from the global surveillance dataset, as this distribution was supported by the observed data for these two variables. For other parameters the uniform distribution was used, to better account for the uncertainty around estimates based on few observations (those from the supplementary dataset) and around the CDRs for each country; the limits of the uniform distributions were based on the most extreme observations in the supplementary dataset and the limits estimated by the WHO for the CDRs. Uncertainty intervals for our results were then sampled from the 2.5th and 97.5th percentile of the distributions for the estimates for each country, and point estimates from the median of all estimates for the country. These were then summed across each of the six WHO regions in order to provide regional estimates, which were rounded to the nearest 1000.

The equation used for estimation in countries with complete data on notifications for the 15-24-year-old age group was:

$$
\mathrm{TB}_{15-19}=\left(\mathrm{N}_{15-24} \times \mathrm{P}_{15-19}\right) \times(1 / \mathrm{CDR})
$$

where:

$$
\mathrm{N}_{15-24}=\left(\mathrm{SP}_{15-24}+\mathrm{SN}_{15-24}+\mathrm{EP}_{15-24}\right)
$$

\begin{tabular}{|c|c|c|}
\hline Parameter & Definition & $\begin{array}{l}\text { Assumed } \\
\text { distribution }\end{array}$ \\
\hline$P_{10-14}$ & $\begin{array}{l}\text { Proportion of tuberculosis notifications among } 0-14 \text { year olds } \\
\text { contributed by the } 10-14 \text {-year-old age group }\end{array}$ & Uniform \\
\hline$P_{15-19}$ & $\begin{array}{l}\text { Proportion of tuberculosis notifications among } 15-24 \text { year olds } \\
\text { contributed by the } 15-19 \text {-year-old age group }\end{array}$ & Uniform \\
\hline$P_{20-24}$ & $\begin{array}{l}\text { Proportion of tuberculosis notifications among } 15-24 \text { year olds } \\
\text { contributed by the } 20-24 \text { year-old age group }\end{array}$ & Uniform \\
\hline $\boldsymbol{\beta}$ & $\begin{array}{l}\text { Regression coefficient for the increase in the proportion of all } \\
\text { sputum smear-positive notifications pertaining to the } 15- \\
24 \text {-year-old age group per } 1 \% \text { increase in the proportion of the } \\
\text { source population in this age group }\end{array}$ & Normal \\
\hline C & Constant term from linear regression model for $\mathrm{P}(\mathrm{SP})_{15-24}$ & \\
\hline$P(S P)_{15-24}$ & $\begin{array}{c}\text { Proportion of all smear-positive tuberculosis notifications } \\
\text { contributed by the } 15-24 \text {-year-old age group }\end{array}$ & \\
\hline $\mathbf{R}_{\text {(SN/EP-TB):(SP-TB) }}$ & $\begin{array}{c}\text { Ratio of smear-negative (including smear not done)/extrapulmonary } \\
\text { tuberculosis notifications to smear-positive tuberculosis } \\
\text { notifications in the } 15-24 \text {-year-old age group }\end{array}$ & Normal \\
\hline CDR & Case detection rate for tuberculosis in the country as of 2016 & Uniform \\
\hline
\end{tabular}


The equation for $\mathrm{SN}_{15-24}$ and $\mathrm{EP}_{15-24}$ in countries missing data on those variables was:

$$
\mathrm{SN}_{15-24}+\mathrm{EP}_{15-24}=\mathrm{SP}_{15-24} \times \mathrm{R}_{(\mathrm{SN} / \mathrm{EP}-\mathrm{TB}):(\mathrm{SP}-\mathrm{TB})}
$$

The equation for $\mathrm{SP}_{15-24}$ in countries missing data for that variable was:

$$
\mathrm{SP}_{15-24}=\text { All SP-TB } \times \mathrm{P}(\mathrm{SP})_{15-24}
$$

where:

$$
\mathrm{P}(\mathrm{SP})_{15-24}=\mathrm{C}+\beta \times\left(\text { population }_{15-24} / \text { population }_{\text {all ages }}\right)
$$

\section{Results}

We estimate that 1.78 million (UI 1.23-3.00 million) adolescents and young adults aged 10-24 years developed tuberculosis during 2012. Estimated case numbers were lowest in the 10-14-year-old age group and highest in the 20-24-year-old age group. Figure 1 shows estimates with uncertainty intervals for 5 -year age bands by WHO region. The WHO South East Asian Region and the WHO African Region had the highest total caseloads (table 2).

The observed proportion of all tuberculosis in the 0-14-year-old age group which affected young adolescents aged 10-14 years ranged from 0.107 in South Africa to 0.426 in Brazil, while the proportion of all tuberculosis in the 15-24-year-old age group that affected the 15-19-year-old age group in the supplementary data ranged from 0.298 in Estonia to 0.376 in Indonesia (supplementary table S2).

Visual inspection via scatter plot confirmed that the relationship between the proportion of the population aged 15-24 years and the proportion of smear-positive tuberculosis notifications that pertain to 15-24 year olds in the global dataset was linear in nature and the $\mathrm{R}^{2}$-value indicated that variation in the former variable explained $34.7 \%$ of variation in the latter. According to the linear model, as the percentage of the population aged 15-24 years increases $1 \%$, the proportion of all smear-positive tuberculosis notifications that pertain to that age group increases by $1.16 \%$ (95\% CI 1.09-1.23). This value was used to estimate the expected number of smear-positive tuberculosis notifications in the 15-24-year-old age group for seven countries that did not report this figure.

The observed ratio of smear-negative and extrapulmonary notifications to smear-positive notifications among the 15-24-year-old age group was $1.28: 1$ (95\% CI 1.15-1.42). This figure was used to estimate the expected number of smear-negative and extrapulmonary notifications for the 15-24-year-old age group for 50 countries that did not report this figure. Of these countries, 27 were in the WHO African Region, with the remainder distributed across the other five WHO regions (supplementary table S1).

\section{Discussion}

This study provides original global estimates of the large burden of tuberculosis in adolescents and young adults aged 10-24 years. We estimate that 1.78 million young people developed tuberculosis worldwide in 2012. Numbers of cases increase markedly by age group across adolescence and into young adulthood,

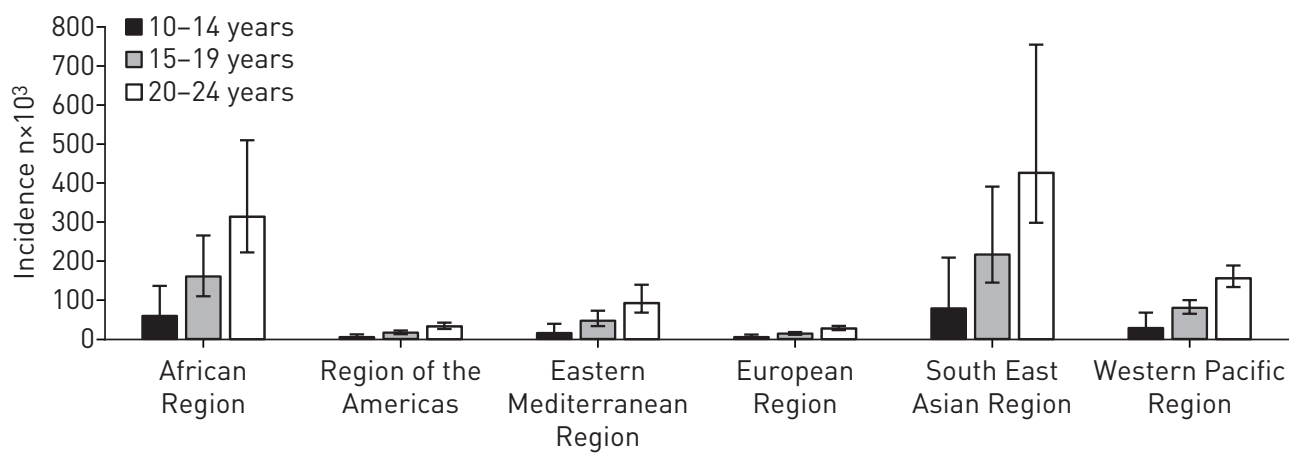

FIGURE 1 Estimated tuberculosis incidence for adolescents and young adults, by 5 -year age band and World Health Organization region, 2012. Vertical lines represent uncertainty intervals around best estimates of tuberculosis incidence. 
TABLE 2 Estimates of new cases of tuberculosis among adolescents and young adults aged 10-24 years, by World Health Organization region, 2012

\begin{tabular}{lcccc} 
& \multicolumn{4}{c}{ Age group years } \\
\cline { 2 - 5 } & $\mathbf{1 0 - 1 4}$ & $\mathbf{1 5 - 1 9}$ & $\mathbf{2 0 - 2 4}$ & $\mathbf{1 0 - 2 4}$ \\
\hline African Region & $59(27-136)$ & $161(109-266)$ & $314(222-510)$ & $534(359-912)$ \\
Region of the Americas & $5(2-12)$ & $17(13-22)$ & $33(26-42)$ & $54(41-76)$ \\
Eastern Mediterranean Region & $15(6-39)$ & $47(33-73)$ & $92(68-139)$ & $155(107-251)$ \\
European Region & $5(2-12)$ & $14(11-18)$ & $27(23-34)$ & $46(37-63)$ \\
South East Asian Region & $78(31-209)$ & $217(145-391)$ & $426(298-755)$ & $721(473-1354)$ \\
Western Pacific Region & $28(13-68)$ & $80(65-100)$ & $156(133-189)$ & $265(211-356)$ \\
All regions & $192(82-476)$ & $535(376-869)$ & $1049(770-1668)$ & $1775(1228-3013)$
\end{tabular}

Data are presented as $\mathrm{n} \times 10^{3}$ (uncertainty interval).

with the lowest numbers in 10-14 year olds and the highest numbers in 20-24 year olds. These estimates were determined using the most comprehensive source of data available, i.e. the global tuberculosis surveillance dataset [19], supplemented with data from several large tuberculosis-endemic countries from different epidemiological and geographical contexts that are broadly representative of most settings globally.

The marked increase in the estimated tuberculosis caseload between early adolescence and young adulthood is consistent with historical observation [5], and with age-stratified tuberculosis estimates published by the Global Burden of Diseases group in 2013 [22]. Historical data from the pre-HIV era further confirm that this phenomenon is an independent feature of tuberculosis epidemiology [5]. However, the concomitant increase in HIV prevalence throughout adolescence in high HIV incidence settings [23] likely exacerbates this phenomenon in many modern tuberculosis epidemics, particularly those in the African region. The fact that tuberculosis risk increases so markedly throughout this period suggests that adolescence may be an important window for preventive interventions for tuberculosis.

The requirements for reporting tuberculosis data to the WHO have expanded over time as tuberculosis control strategies have widened beyond an initial focus on those cases most likely to contribute to transmission, i.e. cases of sputum smear-positive tuberculosis. In 2006, the WHO revised tuberculosis registers to record and report all new tuberculosis cases by type, disaggregated by the age groups of $0-4$, 5-14 and $>15$ years, with new sputum smear-positive pulmonary tuberculosis cases in adults to be further disaggregated into 10 -year age groups (i.e. $15-24,25-34, \ldots, \geqslant 65$ years) [24], although as observed during this analysis, implementation by countries varied. In 2013, the WHO requested that countries transition to reporting all new and relapse tuberculosis cases together (regardless of site of disease), disaggregated by age group $(0-5,5-14,15-24,25-34$ years, etc.) [19]. However, current reporting practices still do not allow direct determination of the numbers of notifications in adolescents and young adults (aged 10-24 years). As case-based electronic tuberculosis surveillance systems are more widely adopted and implemented, more nuanced analysis of tuberculosis notifications in these age groups will become possible in a variety of settings.

There are a number of important limitations to the accuracy of these estimates. Many young people at risk of tuberculosis live in countries with at least some missing data. It was necessary to estimate the expected number of smear-negative and extrapulmonary notifications in the 15-24-year-old age group for 50 countries in total, including several large, high tuberculosis burden countries (supplementary table S1).

We chose to estimate smear-negative and extrapulmonary notifications from smear-positive tuberculosis notifications for several reasons. First, there is greater diagnostic certainty for smear-positive tuberculosis than for the other forms of the disease. Second, all but seven countries reported smear-positive notifications with full age disaggregation in 2012, providing a high-quality indicator of the incidence of this form of disease in the 15-24-year-old age group after adjustment for underreporting. As the relative prevalence of smear-positive disease appears to be largely biological [25], the ratio between forms of disease was relatively constant across countries.

Finally, CDRs in several high tuberculosis burden countries are still based on expert opinion because national tuberculosis prevalence surveys have not yet been completed for all priority countries [21]. CDRs for several large countries have been revised downward in recent years, resulting in higher estimates of the global tuberculosis burden [21]. If case detection in any other large, high tuberculosis burden countries is presently overestimated, then the point estimates of tuberculosis burden given here will be conservative. 
Likewise, if case detection is lower in adolescents than in adults, as has been documented in some settings $[9,26]$, we will also have underestimated the tuberculosis burden in this age group. CDRs may vary with age given the age-related challenges for bacteriological confirmation in young adolescents (aged 1014 years) as well as barriers to health service access for young people in some settings. At present there are no comprehensive global data on age-specific CDRs for tuberculosis, which prevents adjustment for potential age-specific CDRs. An additional challenge is that young adolescents are not usually included in national prevalence surveys. However, as estimates of the uncertainty around each of the key parameters have been incorporated into this analysis, we believe that the likely extent of uncertainty is captured by the intervals around our point estimates.

In conclusion, this study provides original estimates of the large burden of tuberculosis in adolescent and young adults globally in 2012, showing a sharp age-related increase in case numbers. The limitations of the accuracy of these estimates are recognised and highlight the pressing need for further studies of the epidemiology of tuberculosis among young people in a range of settings, and for continued strengthening of tuberculosis surveillance systems. Nonetheless, this work provides an important platform for increasing attention to and advocacy for the needs of this neglected and vulnerable group, who must be considered in scaling up efforts to end the global tuberculosis epidemic.

\section{Acknowledgements}

Age-specific tuberculosis data were provided by the national tuberculosis programmes of Brazil, Indonesia and South Africa. The European Centres for Disease Control provided age-specific data pertaining to Romania and Estonia. Dennis Petrie (Monash University, Clayton, Australia) provided guidance on the generation of confidence intervals.

Author contributions: S.M. Graham and K.J. Snow conceived the study. K.J. Snow conducted the literature search and created the figures. K.J. Snow and C. Sismanidis designed the study and analysed the data. S.M. Graham, S.M. Sawyer and J. Denholm interpreted the data. All authors contributed to writing the manuscript. C. Sismanidis is a staff member of the World Health Organization. The author alone is responsible for the views expressed in this publication and they do not necessarily represent the decisions or policies of the World Health Organization.

\section{References}

1 Sawyer SM, Afifi RA, Bearinger LH, et al. Adolescence: a foundation for future health. Lancet 2012; 379: $1630-1640$.

2 Patton GC, Sawyer SM, Santelli JS, et al. Our future: a Lancet commission on adolescent health and wellbeing. Lancet 2016; 387: 2423-2478.

3 United Nations Population Division. World population prospects, 2015 revision. 2015. http://esa.un.org/unpd/ wpp/DVD/ Date last accessed: November 13, 2017.

4 Marais BJ, Gie RB, Schaaf HS, et al. The clinical epidemiology of childhood pulmonary tuberculosis: a critical review of literature from the pre-chemotherapy era. Int J Tuberc Lung Dis 2004; 8: 278-285.

5 Morabia A. Snippets from the past: cohort analysis of disease rates - another piece in a seemingly still incomplete puzzle. Am J Epidemiol 2014; 180: 189-196.

6 Comstock GW, Livesay VT, Woolpert SF. The prognosis of a positive tuberculin reaction in childhood and adolescence. Am J Epidemiol 1974; 99: 131-138.

7 Mathad JS, Gupta A. Tuberculosis in pregnant and postpartum women: epidemiology, management, and research gaps. Clin Infect Dis 2012; 55: 1532-1549.

8 Rendell NL, Batjargal N, Jadambaa N, et al. Risk of tuberculosis during pregnancy in Mongolia, a high incidence setting with low HIV prevalence. Int J Tuberc Lung Dis 2016; 20: 1615.

9 Nduba V, Hoog AH, Mitchell E, et al. Prevalence of tuberculosis in adolescents, western Kenya: implications for control programs. Int J Infect Dis 2015; 35: 11-17.

10 Enane L, Lowenthal ED, Arscott-Mills T, et al. Loss to follow-up among adolescents with tuberculosis in Gaborone, Botswana. Int J Tuberc Lung Dis 2016; 20: 1320-1325.

11 UNAIDS. Report on the global AIDS epidemic. 2013. www.unaids.org/en/resources/campaigns/globalreport2013/ globalreport Date last accessed: November 13, 2017.

12 World Health Organization. The end TB strategy. 2015. www.who.int/tb/post2015_strategy/en/ Date last accessed: November 13, 2017.

13 World Health Organization. Roadmap for childhood tuberculosis: towards zero deaths. 2013. www.who.int/tb/ areas-of-work/children/roadmap/en/ Date last accessed: November 13, 2017.

14 World Health Organization. Global tuberculosis report. 2012. http://apps.who.int/iris/bitstream/10665/75938/1/ 9789241564502_eng.pdf Date last accessed: November 13, 2017.

15 Dodd PJ, Gardiner E, Coghlan R, et al. Burden of childhood tuberculosis in 22 high-burden countries: a mathematical modelling study. Lancet Global Health 2014; 2: e453-e459.

16 Jenkins HE, Tolman AW, Yuen CM, et al. Incidence of multidrug-resistant tuberculosis disease in children: systematic review and global estimates. Lancet 2014; 383: 1572-1579.

17 World Health Organization. Global tuberculosis report. 2016. http://apps.who.int/iris/bitstream/10665/250441/1/ 9789241565394-eng.pdf Date last accessed: November 13, 2017.

18 World Health Organization. Global tuberculosis database. 2014. www.who.int/tb/publications/global_report/en/ Date last accessed: November 13, 2017.

19 World Health Organization. Global tuberculosis database. 2017. www.who.int/tb/publications/global_report/en/ Date last accessed: November 13, 2017.

20 World Health Organization. Definitions and reporting framework for tuberculosis - 2013 revision. 2014. www. who.int/tb/publications/definitions/en/ Date last accessed: November 13, 2017. 
21 World Health Organization. Global tuberculosis report. 2013. http://apps.who.int/iris/bitstream/10665/91355/1/ 9789241564656 eng.pdf Date last accessed: November 13, 2017.

22 Murray CJ, Ortblad KF, Guinovart C, et al. Global, regional, and national incidence and mortality for HIV, tuberculosis, and malaria during 1990-2013: a systematic analysis for the Global Burden of Disease Study 2013. Lancet 2014; 384: 1005-1070.

23 Idele P, Gillespie A, Porth T, et al. Epidemiology of HIV and AIDS among adolescents: current status, inequities, and data gaps. J Acquir Immune Defic Syndr 2014; 66: S144-S153.

24 World Health Organization. Revised TB recording and reporting forms and registers - version 2006. 2006. www. who.int/tb/err/rr_final_forms_en.pdf Date last accessed: November 13, 2017.

25 World Health Organization. Systematic screening for active tuberculosis: principles and recommendations. 2013. www.who.int/tb/publications/Final_TB_Screening_guidelines.pdf Date last accessed: November 13, 2017.

26 Lestari BW, Arisanti N, Siregar AYM, et al. High caseload of childhood tuberculosis in hospitals on Java island, Indonesia: a cross sectional study. BMC Public Health 2011; 11: 784. 